\title{
INTELIGENCIA EMOCIONAL Y NECESIDAD COGNITIVA EN ESTUDIANTES UNIVERSITARIOS
}

\author{
Luis Miguel Escurra Mayaute \\ Ana Delgado Vásquez
}

\begin{abstract}
RESUMEN
Se analizan las relaciones entre la inteligencia emocional y la necesidad cognitiva, teniendo en cuenta estudiantes del primer semestre de las diversas especialidades de la UNMSM. Los participantes corresponden a una muestra aleatoria de 365 alumnos pertenecientes de manera representativa a las 19 facultades de las cinco áreas de especialización de la universidad. Se encontró diferencias significativas entre varones y mujeres en inteligencia emocional. En la comparación por áreas de especialización se obtuvieron diferencias en empatía, autoconocimiento emocional, automotivación. En cuanto a la necesidad cognitiva no se observaron diferencias significativas en ninguna variable de comparación. Finalmente se obtuvieron correlaciones positivas y estadísticamente significativas entre los constructos estudiados.
\end{abstract}

Palabras clave: Inteligencia emocional, Necesidad cognitiva

\begin{abstract}
It is analized the relationship between emotional intelligence and need for cognition considering Freshmen college students of a public peruvian university (Universidad Nacional Mayor de San Marcos). The participants were 365 students of the 19 schools and five major academic areas. it was found differences among men and women on emotional intelligence. Also, there were differences on specific areas such as empathy, emotional self knowledge, and self motivation. Considering the need for cognition it was found no differences among women and men. Finally, it was found positive correlations between the scores of emotional intelligence and need for cognition.
\end{abstract}

Key Words: Emotional intelligence, need for cognition. 


\section{LA INTELIGENCIA EMOCIONAL}

La Inteligencia Emocional tiene sus raíces en el concepto de "inteligencia social," inicialmente identificado por Thorndike en 1920. Otros autores han identificado otro tipo de inteligencias agrupándolas de la siguiente manera: a) Inteligencia Abstracta, que es la habilidad de entender y manipular símbolos verbales y matemáticos. b) Inteligencia Concreta, que es la habilidad de entender y manipular con objetos. c) Inteligencia social, que es la habilidad de entender y relacionar a las personas (Ruisel, 1992).

La inteligencia social ha sido definida como la habilidad de entender y manejar a los hombres y mujeres, muchachos y muchachas para actuar sabiamente en relaciones humanas.

Gardner (1983) incluye las inteligencias intrapersonal e interpersona1 en su teoría de las inteligencias múltiple. Estos dos tipos de inteligencia serían las que conforman la inteligencia social, las cuales son definidas de la siguiente forma:

a. Inteligencia interpersonal: Es la habilidad de entender a otras personas, identificar lo que los motiva, cómo trabajan, cómo trabajar cooperativamente con ellos.

b. Inteligencia Intrapersonal: Es la habilidad para reconocer los aspectos internos de sí mismo, el acceso a la propia vida emocional, a la propia gama de sentimientos, la capacidad de efectuar discriminaciones entre emociones y ponerles un nombre y recurrir a ellas como un medio de interpretar y orientar la propia conducta.

Según Salovey \& Mayer (1990), la inteligencia emocional, incluye la inteligencia intrapersonal y la inteligencia interpersonal, así como otras habilidades que pueden categorizarse en cinco dominios:

a. Conocimiento de sí mismo: Observándose y reconociendo un sentimiento cuando pasa.

b. Control emocional: Manejando sus sentimientos, de manera que sean apropiados; comprendiendo lo que está detrás de ellos; encontrando maneras de manejar miedos y ansiedades, cólera, y tristeza.

c. Automotivación: Es la capacidad de motivarse a sí mismo, canalizando las mociones en el servicio de una meta.

d. Empatía: Es el reconocimiento de las emociones ajenas, es la sensibilidad para percibir los sentimientos y preocupaciones de los otros, apreciando las diferencias que se presentan en la forma cómo las personas se sienten sobre las cosas.

e. Habilidad para las relaciones interpersonales: Es el control de las emociones en los otros; incluye la competencia social y las habilidades sociales.

El conocimiento de sí mismo (inteligencia del intrapersonal), la empatía y el manejo de las relaciones (inteligencia interpersonal) son esencialmente dimensiones de la inteligencia social.

Goleman (1996) presento su definición de inteligencia emocional basándose en los trabajos desarrollados por Mayer y Salovey (1993). Sin embargo, agregó muchas cosas que él presentó como inteligencia emocional, como es el caso de la inclusión de variables que podrían considerarse más como "rasgos de personalidad" que como componentes de inteligencia emocional. Así por ejemplo, incluyó el optimismo, la perseverancia y la habilidad de retardar la satisfacción como aspectos relevantes de la inteligencia emocional.

En la actualidad existe discordancia acerca de que la inteligencia emocional es más un potencial inherente, o si es un conjunto de "habilidades" o "competencias". Por ejemplo, para Goleman (1997), la inteligencia emocional es básicamente la misma a lo largo de la vida. Mayer y Salovey (1997) han actualizado su definición de inteligencia emocional, 
indicando que involucra la habilidad de percibir con precisión, estimar, y expresar las emociones; la habilidad de acceder y/o generar sentimientos cuando ellos facilitan el pensamiento; la habilidad de entender la emoción y el conocimiento emocional; y la habilidad de regular las emociones para promover crecimiento emocional y intelectual.

El campo de estudio de la inteligencia emocional, presenta dos afrontes generales. El primer afronte es el socio-emocional que incluye habilidades así como una serie de rasgos de personalidad. Esta concepción fue popularizada por el libro de Goleman (1996). En alguna magnitud, el modelo de Salovey y Mayer (1990) es también un modelo socioemocional o mixto. El segundo afronte corresponde a un modelo de habilidad. Este modelo de inteligencia emocional establece un eslabón entre las emociones y el razonamiento.

El modelo de habilidad de inteligencia emocional, como el desarrollado por Mayer y Salovey (1997), presenta a la inteligencia emocional como un juego de habilidades que combinan emociones y cognición. Ellos definieron la inteligencia emocional como la habilidad de percibir emociones, acceder y generar emociones para ayudar al pensamiento, entender las emociones, aumentar el conocimiento emocional, y regular las emociones reflexivamente para promover el crecimiento emocional y intelectual (Mayer \& Salovey, 1997). Específicamente, la inteligencia emocional está compuesta por cuatro componentes separados:

a. Identificación de Emociones; Es la habilidad de reconocer en uno y en los demás como se sienten.

b. Utilización de Emociones: Es la hab5lidad para generar emociones y razonar con esta emoción.

c. Comprensión de las Emociones: Es la habilidad para entender las emociones complejas y las "cadenas" de emociones, cómo la transición de emociones de una fase a otra,

d. Dirección de las Emociones; referida a la habilidad para manejar las emociones en su ego y en otros.

\section{LA NECESIDAD COGNITIVA}

La necesidad cognitiva es el elemento impulsor y energético para el desencadenamiento de un comporta-miento, que se manifiesta en la vida diaria en la predisposición de los sujetos a organizar activamente la información de su entorno, de manera que está permanentemente a la búsqueda de los estímulos de su medio ambiente. Así por ejemplo, en el caso de estudiantes del nivel de educación superior universitaria, la necesidad cognitiva puede expresarse en términos de la búsqueda de nueva información con respecto al campo profesional de estudio, así como sus actitudes hacia el mismo.

El estudio de la necesidad cognitiva ha recibido el aporte teórico y práctico de diversos autores, destacando entre ellos Cacioppo y Petty (1982), quienes elaboraron una escala para cuantificada. En nuestro medio, Delgado (1997) realizó la adaptación de la Escala de Necesidad Cognitiva en una muestra de alumnos del I semestre académico de la U.N.M.S.M.

Delgado (1997) estudió la relación entre la Necesidad Cognitiva y las Dimensiones de Personalidad de Eysenck en estudiantes I semestre académico de la U.N.M.S.M., encontrando que los participantes tendían a presentar una mayor necesidad cognitiva, lo que indicaría que estos alumnos poseen una elevada predisposición para el estudio, para conocer cosas nuevas y para enfrentarse a diversos tipos de problemas, lo cual es esperable y deseable en todos aquellos que están iniciando estudios superiores. Asimismo, se encontró que los niveles de necesidad cognitiva no varían en función al sexo, es decir, los universitarios varones y mujeres presentan por igual este tipo de motivación por conocer las cosas. 
En la comparación de los niveles de necesidad cognitiva en función al área de estudio, se encontró que no existen diferencias estadísticamente significativas, 10 que indica que en independencia del tipo de estudio profesional que les interesa desarrollar a los alumnos, éstos tienen una adecuada motivación en general hacia el estudio.

El análisis de las correlaciones permitió observar que en algunos casos existían relaciones altas y estadísticamente significativas entre la necesidad cognitiva y las dimensiones básicas de la personalidad en estudiantes del I semestre académico de la UNMSM, encontrándose específicamente que a mayor necesidad cognitiva mayor nivel de Extraversión. Así mismo, se encontró una correlación negativa y significativa entre la necesidad cognitiva y la dimensión de Dureza. También se observó que no existía una correlación estadísticamente significativa entre la necesidad cognitiva y el nivel de Emocionalidad.

\section{VARIABLES DE ESTUDIO}

Dado que se trata de un estudio correlacional, las variables estudiadas fueron las siguientes:

a. Inteligencia Emocional: Evaluada con los puntajes de la prueba de Inteligencia Emocional.

b. Necesidad Cognitiva: Evaluada a través de los puntajes totales de la Escala de Necesidad Cognitiva.

Adicionalmente se realizaron comparaciones según las siguientes variables:

a. Sexo: se consideraron estudiantes universitarios, varones y mujeres.

b. Especialidad: Fue determinada a través de la clasificación de las Facultades elaborada por la U.N.M.S.M. y que incluye las áreas: A, B, C, D y E, las que se detallan a continuación:

Área A: Química, Ciencias Biológicas, Física, Matemática, Estadística, Investigación Operativa.

Área B: Medicina Humana, Farmacia y Bioquímica, Odontología, Medicina Veterinaria, Obstetricia, Enfermería, Laboratorio Clínico y Anatomía Patológica, Terapia Física y Rehabilitación, Radiología, Terapia Ocupacional, Nutrición, Psicología.

Área C: Ingeniería Mecánica de Fluidos, Ingeniería Geológica, Ingeniería Geográfica, Ingeniería de Minas, Ingeniería Metalúrgica, Ingenie-ría Industrial, Ingeniería Electrónica, Ingeniería Química, Ingeniería de Sistemas, Ingeniería Eléctrica.

Área D: Contabilidad, Economía, Administración, Turismo, Negocios Internacionales.

Área E: Derecho y Ciencias Políticas, Filosofía, Linguiística, Comunicación Social, Arte, Bibliotecología y Ciencias de la Información, Historia, Sociología, Antropología, Arqueología, Trabajo Social, Geografía, Literatura, Educación y Educación Física.

\section{HIPÓTESIS}

\section{Hipótesis General}

"En los estudiantes del primer semestre de las diversas especialidades profesionales de la UNMSM se presentan relaciones estadísticamente significativas entre la inteligencia emocional y la necesidad cognitiva ".

\section{Hipótesis Específicas}

$\mathbf{H}_{1}$ : "En los estudiantes del 1 semestre académico de la UNMSM la inteligencia emocional varía en función al sexo".

$\mathbf{H}_{2}$ : "En los estudiantes del 1 semestre académico de la UNMSM la inteligencia emocional varía en función al tipo o área de especialidad".

$\mathbf{H}_{3}$ : "En los estudiantes del 1 semestre académico de la U.N.M.S.M. los niveles de 
necesidad cognitiva varían en función al sexo".

$\mathbf{H}_{4}$ : "En los estudiantes del 1 semestre académico de la UNMSM los niveles de necesidad cognitiva varían en función al tipo de especialidad o área de estudio".

$\mathbf{H}_{5}$ : "Existen correlaciones altas y estadísticamente significativas entre la variable inteligencia emocional y la variable necesidad cognitiva en los estudiantes del 1 semestre académico de la UNMSM".

\section{METODO}

El presente trabajo siguiendo a Kerlinger (1988) constituye una investigación correlacional, pues los resultados obtenidos en la medición de las variables han sido correlacionados, para determinar el grado de asociación existente entre los aspectos estudiados (Van Dalen y Meyer, 1971; Alarcón, 1991; Hernández, F ernández y Baptista, 1991; Sánchez y Reyes, 1998). Adicionalmente, se realizaron comparaciones de acuerdo al sexo y el área de especialidad con la finalidad de establecer con mayor precisión las características de los constructos estudiados.

\section{Participantes.}

El tamaño de la muestra de investigación se estableció a través del programa informático SOTAM, el cual determinó que debían considerarse 375 participantes, los cuales fueron seleccionados considerando la proporción de alumnos por área y por escuela académicoprofesional.

Instrumentos de Recolección de Datos. Prueba de Inteligencia Emocional, fue construida por Escurra (1999), mide la habilidad de percibir con precisión, estimar, y expresar las emociones; la habilidad de acceder y/o generar sentimientos cuando ellos facilitan el pensamiento; la habilidad de entender la emoción y el conocimiento emocional; y la habilidad de regular las emociones para promover el crecimiento emocional y intelectual. La prueba está conformada por 68 ítemes que se presentan en un formato tipo Likert de 5 puntos de calificación. La prueba evalúa los siguientes aspectos:

a. Autoconocimiento emocional: Mide la habilidad para reconocer sus propios sentimientos, pudiendo diferenciarlos entre sí. Está conformado por 7 ítemes.

b. Control Emocional: Evalúa la habilidad para controlar los sentimientos; comprendiendo lo que está detrás de ellos, así como las maneras de manejar los miedos, ansiedades, cóleras y tristezas. Está conformado por 15 ítemes.

c. Automotivación: Mide la habilidad del sujeto para motivarse a sí mismo y encausar sus emociones en el servicio de una meta, de manera que el control emocional; permite posponer la satisfacción de los impulsos. Está constituido por 23 ítemes.

d. Empatía: Evalúa la habilidad para reconocer las emociones ajenas, es decir la sensibilidad para asumir los sentimientos y preocupaciones que presentan los demás. Está conformado por 6 Ítemes

e. Habilidad para las relaciones interpersonales: Mide la habilidad para dirigir las emociones, la competencia social y las habilidades sociales. Está constituida por 17 ítemes.

La prueba originalmente estuvo constituida por 106 ítemes los cuales a través de sucesivos análisis de ítemes para cada dimensión permitieron seleccionar los 68 reactivos más discriminativos

El análisis de la confiabilidad por consistencia interna realizado a través del coeficiente Alfa de Cronbach, permitió observar que los resultados fluctuaron entre 0.81 y 0.87 , lo que permitió demostrar que la prueba permitía obtener puntajes confiables. 
La validez se estudió de dos maneras, en primer lugar se trabajó la Validez de Contenido a través del método del criterio de jueces, estableciéndose que los ítemes correspondían al universo de contenido que se trataba de estudiar, obteniéndose coeficientes $\mathrm{V}$ de Aiken significativos.

En segundo lugar se estudio la Validez de Constructo por medio del análisis factorial exploratorio, computado a través del procedimiento de los componentes principales y el método de rotación oblicua Oblimin, encontrándose que existió un solo factor, que explicaba el $72.10 \%$ de la varianza total, lo que corroboró que la prueba de Inteligencia Emocional presentaba Validez de Construcción.

Adicionalmente se trabajó el Análisis Factorial Confirmatorio, aplicando el programa Amos 4.0, demostró que el modelo propuesto de un solo factor presenta en el test de Bondad de Ajuste Chi-Cuadrado (Chmin) un valor de 0.21, el cual con 2 grados de libertad alcanzó una probabilidad de .900 , lo que nos indicó que el modelo era adecuado, notándose además que dicho modelo fue superior al modelo Saturado y al Modelo Independiente, lo que se corroboró en los estadísticos de análisis de residuales (RMR) y los análisis complementarios de bondad de Ajuste como son el GFI y el AGFI. Los cuales certificaron que la prueba presentó validez de Construcción.

Escala de Necesidad Cognitiva (ENC) Desarrollada por Cacioppo y Petty, adaptada por J. Gutiérrez et al. (1993), está conformada por 18 afirmaciones presentadas de forma tanto positiva como negativa con la finalidad de evitar la deseabilidad social. Cada afirmación debe ser calificada por el examinado en una escala de 6 puntos según el grado con que concuerden sus ideas con el enunciado indicado.

Gutiérrez et AI.(1993), evaluaron la confiabilidad a través de la consistencia interna utilizando el método de la división por mitades (par-impar) empleando el coeficiente de corrección de Spearman-Brown, el cual fue aplicado a la escala en general y a las 4 subescalas, los resultados indicaron que la escala global alcanzaba un valor de 0.69 , la subescala de anticipación 0.77 , la de resolución de problemas y la de activación alcanzaron un valor de 0.78 y en la sub-escala de implicación personal se obtuvo 0.47 . Estos resultados han sido considerados, por sus autores como indicadores de una con fiabilidad aceptable.

La validez fue evaluada a través del procedimiento de validez predictiva, pues se trató de verificar si la prueba era capaz de diferenciar entre quienes tenían un alto nivel de necesidad cognitiva (los estudiantes universitarios) y los que tenían una baja necesidad cognitiva (los reclusos). Los resultados indicaron que tanto el puntaje total como los obtenidos en las sub-escalas permitían diferenciar con claridad ambos grupos, por lo cual se concluyó que la escala tiene validez predictiva, a través del método de diferenciación de grupos.

Delgado (1997), realizó la adaptación de la Escala de Necesidad Cognitiva en una muestra de estudiantes del I semestre académico de la UNMSM, en la que se estableció luego del análisis de ítemes correspondiente, que la nueva escala estaba constituida por 12 ítemes, los cuales alcanzaron un coeficiente de confiabilidad de 0.89 . Por otro lado, el análisis de la estructura factorial del constructo que mide la nueva Escala de Necesidad Cognitiva, permitió apreciar al igual que en los estudios de Furguson, Chung y Weigold (1985; en Gutiérrez, Bajén, Sintas y Amat, 1993), que existen 4 factores latentes, los cuales varían en su conformación de estudio a estudio.

Los factores obtenidos en este estudio, están directamente relacionados con la Anticipación Motivadora, la Activación Cognitiva en General, la Activación Cognitiva Simplificada y la Necesidad de Resolver Problemas Abstractos e Intelectuales.

La evaluación global de estos resultados permitió concluir que la Escala de Necesidad Cognitiva Revisada por Delgado (ENC-RD) es un instrumento válido y que permite obtener puntajes confiables para la obtención de información importante sobre el constructo necesidad cognitiva en nuestro medio. 


\section{Procedimiento de Recolección de Datos}

Para cada área de especialización, se elaboró la lista de Facultades que las constituyen, posteriormente en cada Facultad se confeccionó el listado de Escuelas Académico Profesionales que la conformaban, así como los turnos de clases implementados para los alumnos ingresantes. De acuerdo a los resultados obtenidos, se procedió a seleccionar de forma aleatoria las aulas a ser evaluadas.

Una vez seleccionadas las aulas, se efectuaron las coordinaciones necesarias para aplicar las pruebas. En cada aula se evaluó a todos los alumnos presentes, luego se eligieron al azar aquellos casos que pasaron a conformar la muestra de estudio de acuerdo a la proporción de casos correspondiente a cada facultad.

\section{RESULTADOS}

El estudio de la distribución de los puntajes que presentaban los datos, aplicándose la prueba de bondad de ajuste a la curva normal de Kolmogorov-Smirnov, los resultados demostraron (ver Cuadro 1), que todas las variables no fueron significativas, lo que nos permite concluir que presentaban una adecuada aproximación a la curva normal y por lo tanto aplicar estadísticas paramétricas para el análisis de los datos.

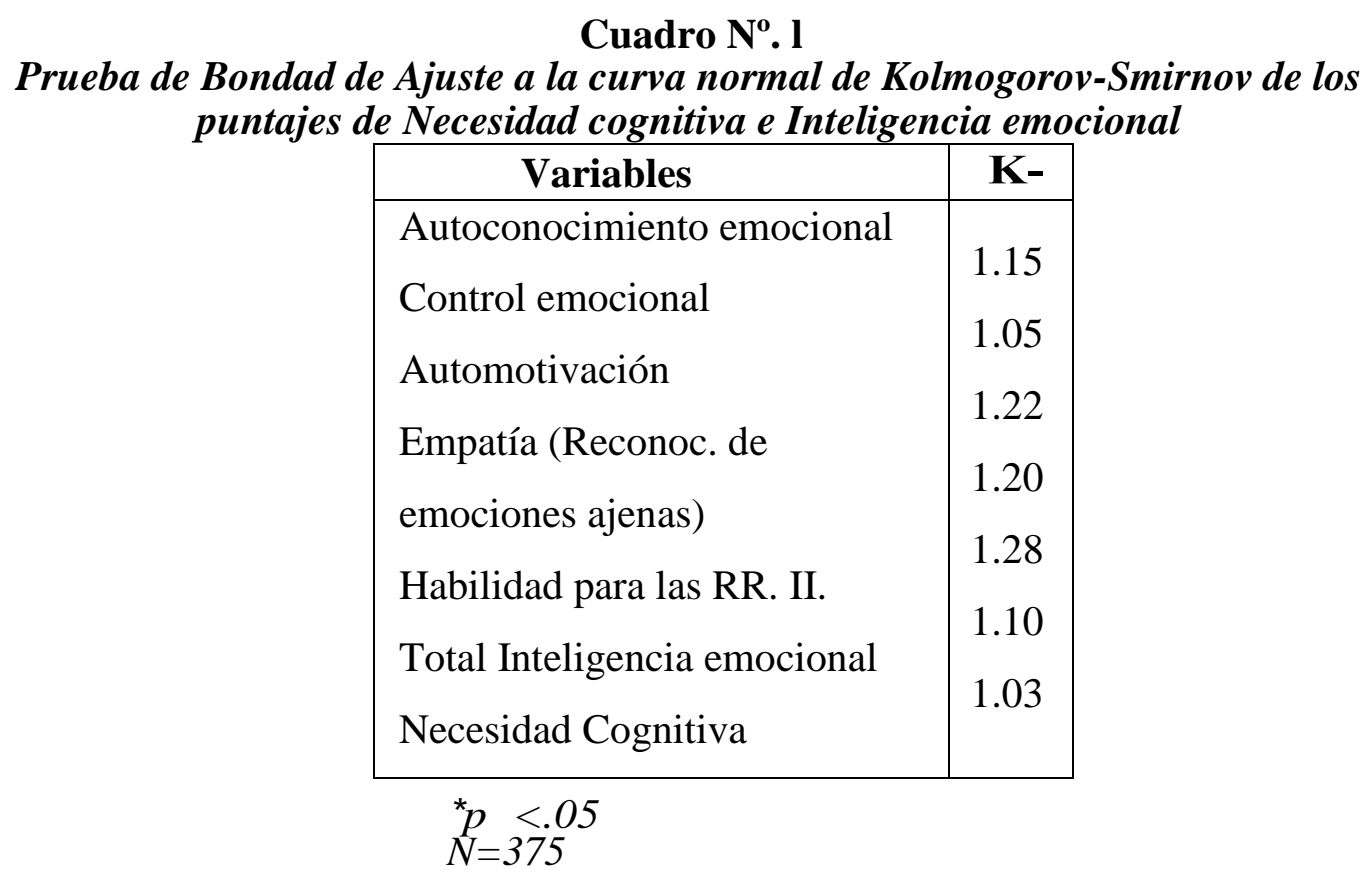

En lo que concierne a las comparaciones por sexo en el cuadro 2, en la inteligencia emocional, se encuentra que sólo existen diferencias significativas en el área de Empatía o Reconocimiento de las emociones ajenas $(\mathrm{Z}=-4.56, \mathrm{p}<.05)$, dándose el caso que las mujeres $(\mathrm{M}=22.55$, D.E. $=3.47)$ alcanzan valores más elevados que los varones $(\mathrm{M}=$ 20.89 , D.E. = 3.58). En tanto que en la necesidad cognitiva no existen diferencias estadísticas significativas entre alumnos y alumnas. 
Cuadro No. 2

Prueba z de comparación de medias de los puntajes de Necesidad cognitiva e Inteligencia emocional según sexo

\begin{tabular}{|c|c|c|c|c|c|}
\hline \multirow[t]{2}{*}{ Variables } & \multicolumn{2}{|c|}{$\begin{array}{c}\text { Masculino } \\
\mathrm{N}=195\end{array}$} & \multicolumn{2}{|c|}{$\begin{array}{c}\text { Femenino } \\
N=180\end{array}$} & \multirow[t]{2}{*}{$\mathbf{Z}$} \\
\hline & $\mathbf{M}$ & $D R$ & $\mathbf{M}$ & $D R$ & \\
\hline & 25.6 & \multirow{4}{*}{5.25} & \multirow{4}{*}{24.67} & \multirow{4}{*}{4.97} & \multirow{4}{*}{1.91} \\
\hline & 8 & & & & \\
\hline Autoconocimiento Emocional & 51.6 & & & & \\
\hline Control emocional & 8 & & & & \\
\hline Automotivación & 82.9 & 6.51 & 52.13 & 6.85 & -0.65 \\
\hline Empatía (Reconocimiento de & 7 & 9.98 & 84.95 & 9.99 & -1.91 \\
\hline 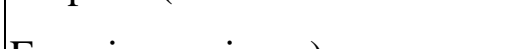 & & 3.58 & 22.55 & 3.47 & -4.56 \\
\hline (Emociones ajenas) & 20.0 & 6.64 & 57.08 & 7.69 & ** \\
\hline Habilidad para las Relaciones & 9 & 267 & 2413 & 253 & 040 \\
\hline interpersonales & 56.7 & 20.1 & 241.5 & $2 \mathrm{~J} . J$ & -0.40 \\
\hline Total Inteligencia emocional & 8 & 60 & 5207 & 0 & -1.25 \\
\hline Necesidad Cognitiva & 238.05 & 0.00 & (2) & 0.21 & 1.11 \\
\hline & 54.2 & & & & \\
\hline & 7 & & & & \\
\hline
\end{tabular}

Respecto a la comparación entre las áreas de estudio, en el cuadro 3, se encuentra que en la variable inteligencia emocional existen diferencias estadísticas significativas en el Autoconocimiento Emocional $(\mathrm{F}(4.379)=1.56, \mathrm{p}>.05)$, presentando los alumnos del área "E" $(\mathrm{M}=27.57)$ valores más elevados que los alumnos de las áreas "B" $(\mathrm{M}=23.94)$, "C" $(\mathrm{M}=24.50)$ y "D" $(\mathrm{M}=24.70)$. Además se observa diferencias en la Automotivación $(\mathrm{F}(4.379)=3.48, \mathrm{p}>.05)$, presentando los alumnos del área " $\mathrm{E} "(\mathrm{M}=86.49)$ valores más elevados que los alumnos del área "C" $(\mathrm{M}=81.79)$. En tanto que también se aprecian diferencias en el total de inteligencia emocional $(\mathrm{F}(4.379)=3.99, \mathrm{p}>.05)$, presentando los alumnos del área "E" $(\mathrm{M}=247.79)$ valores más elevados que los alumnos del área $\mathrm{C}(\mathrm{M}=$ 234.27). Mientras que no existen diferencias significativas en la necesidad cognitiva $(\mathrm{F}(4.379)=1.56, \mathrm{p}>.05)$,

\section{Cuadro $\mathbf{N}^{\circ} 3$}

Análisis de varinaza de un factor de los puntajes de Necesidad cognitiva e Inteligencia emocional según area de estudio

\begin{tabular}{|c|c|c|c|c|c|c|}
\hline \multirow[t]{2}{*}{ Variables } & Area A & Area B & $\begin{array}{l}\text { Area } \\
\text { C } \\
\end{array}$ & $\begin{array}{l}\text { Area } \\
\text { D }\end{array}$ & Area I & $\mathbf{F}$ \\
\hline & $\mathbf{M}$ & $\mathbf{M}$ & $\mathbf{M}$ & M & $\mathbf{M}$ & \\
\hline $\begin{array}{l}\text { Autoconocimiento Emocional } \\
\text { Control emocional }\end{array}$ & $\begin{array}{l}24.77 \\
53.95\end{array}$ & $\begin{array}{l}23.94 \\
51.05\end{array}$ & $\begin{array}{l}24.50_{b} \\
51.23\end{array}$ & $\begin{array}{l}24.70_{c} \\
51.35\end{array}$ & $\begin{array}{l}27.57 \mathrm{a} \\
\mathrm{bc}\end{array}$ & $\begin{array}{l}7.36^{* * * *} \\
2.33\end{array}$ \\
\hline
\end{tabular}




\begin{tabular}{l|l|l|l|l|l|l|}
\hline Automotivación & 86.82 & 82.95 & 81.79 & 83.99 & 53.22 & $3.48 * *$ \\
Empatia (reconocimiento de emociones & 21.64 & 22.17 & 20.79 & 21.94 & 86.49 & 2.47 \\
ajenas) & 57.50 & 56.78 & 55.96 & 56.56 & 22.11 & 1.44 \\
Habilidad para las relaciones & 244.68 & 236.89 & 234.2 & 238.5 & 58.30 & $3.99 * *$ \\
interpersonales & 55.09 & 54.73 & $7 \mathrm{a}$ & 5 & 247.7 & 1.56 \\
Total Inteligencia Emocional & & & 52.84 & 53.08 & $9 \mathrm{a}$ & \\
Necesidad Cognitiva & & & & 53.0 & \\
& \\
*p<.05 **p<.01 ***p<.0001 & & & & \\
G.L. $1=4$ \\
G.L. $2=370$ \\
N 375
\end{tabular}

\section{Comparaciones significativas a través de la prueba de Scheffé: a. b. e}

El análisis de correlaciones entre las áreas de las prueba de Inteligencia Emocional y de Necesidad Cognitiva, permite observar que existen correlaciones positivas y significativas, como las del Autoconocimiento emocional $(r=0.64)$, Automotivación $(r=0.66)$, Control Emocional $(\mathrm{r}=0.68)$ y Habilidad para las relaciones interpersonales $(\mathrm{r}=0.67)$ serían clasificadas como correlaciones moderadas, en tanto que con Empatía o Reconocimiento de las emociones ajenas $(\mathrm{r}=0.76)$ y el puntaje total de la Inteligencia Emocional $(\mathrm{r}=0.78)$, podrían clasificarse como correlaciones altas.

\section{Cuadro No4}

Matriz de correlaciones entre necesidad cognitiva e inteligencia emocional

\begin{tabular}{|c|c|}
\hline $\begin{array}{rr}\text { Variables } & \text { Necesidad cog } \\
\mathbf{R}\end{array}$ & nitiva \\
\hline Autoconocimiento emocional & $0.64 * * *$ \\
\hline Control emocional & $0.68 * * *$ \\
\hline Automotivación & $0.66 * * *$ \\
\hline Empatía (recon. emociones ajenas) & $0.76 * * *$ \\
\hline Habilidad para RR.II & $0.67 * * *$ \\
\hline Total Inteligencia Emocional & $0.78 * * *$ \\
\hline
\end{tabular}

El análisis de la estructura latente que existe entre las variables estudiadas realizado a través del análisis factorial en base al procedimiento de reducción de los componentes principales y al método de rotación Varimax (Ver cuadro 5) indican que existe entre ellos un solo factor que permite explicar el $76.20 \%$ de la Varianza total. Estos hallazgos son validados por el hecho que la matriz de correlación presenta un test de esfericidad de Bartlett que es significativo $(768.17, \mathrm{p}<.05)$ Y por el elevado valor del test de Adecuación del Muestreo de KaiserMeyer-Olkin (0.89). 
Cuadro No. 5

Análisis Factorial de los puntajes de Inteligencia emocional y Necesidad cognitiva

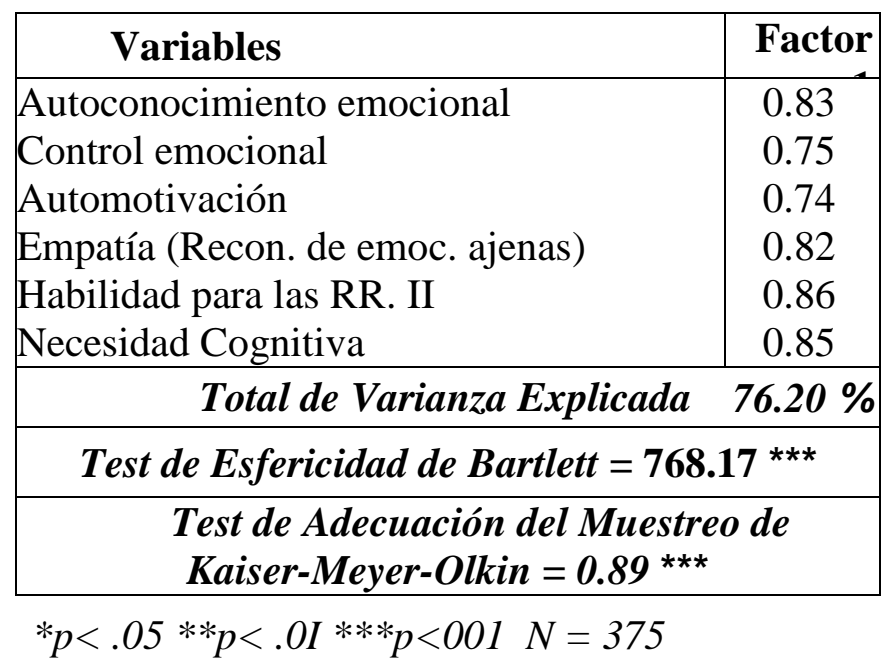

El análisis jerárquico de las áreas de la prueba de Inteligencia Emocional (Ver Cuadro 6), indica que el valor más elevado corresponde a la Automotivación $(\mathrm{M}=3.65$, D.E. $=0.44)$, seguido por Empatía o reconocimiento de emociones ajenas $(\mathrm{M}=3.61$, D.E.=0.60), Autoconocimiento emocional $(\mathrm{M}=3.60$, D.E. $=0.73)$, Control emocional $(\mathrm{M}=3.46$,

D.E. $=0.45)$ y Habilidad para las relaciones interpersonales $(\mathrm{M}=3.35$, D.E. $=0.42)$.

Cuadro No. 6 Orden jerárquico de las áreas de la Prueba de Inteligencia emocional

\begin{tabular}{|l|c|c|c|}
\hline $\mathbf{O}$ & Variables & M & D.E. \\
\hline 1 & Automotivación & 3.65 & 0.44 \\
2 & Empatía (Recon. & 3.61 & 0.60 \\
3 & deemoc.ajenas & 3.60 & 0.73 \\
4 & Autoconocimiento emocional & 3.46 & 0.45 \\
5 & Control emocional & 3.35 & 0.42 \\
\hline
\end{tabular}

\section{DISCUSIÓN}

En primer lugar se encuentra que tanto las distribuciones de los puntajes de las escalas de inteligencia emocional y la necesidad cognitiva, se aproximan a la curva normal. Estos hallazgos indican que los constructos estudiados presentan valores extremos en menor proporción y una mayor cantidad de casos en los valores centrales, lo cual nos indica la diversidad de puntuaciones encontradas en los participantes.

Otros hallazgos importantes corresponden a las comparaciones por sexo, pues los varones y las mujeres presentan niveles. similares de necesidad cognitiva, lo cual corrobora los hallazgos obtenidos por Delgado (1997) en un estudio previo. Situación que se presenta de forma similar en el caso de la inteligencia emocional en los aspectos de Autoconocimiento emocional, Control Emocional, Automotivación, Habilidades para las Relaciones Interpersonales, así como en el total de inteligencia emocional. Aunque es necesario destacar que las mujeres presentan un mayor nivel de empatía que los varones, lo cual estaría asociado al tipo de rol que ejercen en la sociedad, que les lleva a interactuar y tratar 
de comprender a los demás, es decir que las mujeres son más sensibles y capaces de reconocer las emociones ajenas que los varones.

En lo que concierne al análisis de acuerdo a las áreas académicas, encontramos que

Un hallazgo relevante corresponde a la existencia de correlaciones positivas y estadísticamente significativas entre la necesidad cognitiva y todas las áreas de la inteligencia emocional, lo cual nos indicaría que ambos constructos estarían asociados o vinculados entre si, de manera que a mayor necesidad cognitiva el sujeto tiene mayor posibilidad de captar lo que ocurre en su entorno y ello podría facilitar el proceso de interacción social que se genera en el contexto de la inteligencia emocional. Lo cual es corroborado con el análisis factorial que nos permitió observar que la necesidad cognitiva y las áreas de la inteligencia emocional constituyen una sola dimensión, es decir que ambos son elementos que constituyen parte de la personalidad.

El hecho que en el estudio de la inteligencia emocional, el aspecto de la automotivación es el que obtiene la puntuación más elevada, implica que los alumnos tiene capacidad para motivarse a sí mismo y encausar sus emociones en el servicio de una meta, de manera que el control emocional; les permite posponer la satisfacción de los impulsos, lo cual era de esperarse en el caso de los participantes del estudio. 


\section{CONCLUSIONES}

1. Las distribuciones de los puntajes de las escalas de inteligencia emocional y la necesidad cognitiva, se aproximan a la curva normal.

2.Los varones y las mujeres presentan niveles similares de necesidad cognitiva y de inteligencia emocional en los aspectos de autoconocimiento emocional, control emocional, automotivación, habilidades para las relaciones interpersonales. Y total inteligencia emocional.

3. Las mujeres presentan un mayor nivel de empatía que los varones.

4. Las áreas académicas presentan niveles similares en necesidad cognitiva y la inteligencia emocional en los aspectos de: control emocional, empatía, habilidades para las relaciones interpersonales.

5. Los alumnos pertenecientes al área $\mathrm{E}$ alcanzan mayores niveles de autoconocimiento emocional que los alumnos de las áreas B, C, y D.

6. Los alumnos pertenecientes al área E alcanzan mayores ni veles de automotivación que los alumnos del área $\mathrm{C}$.

7. Los alumnos pertenecientes al área $\mathrm{E}$ alcanzan mayores niveles de inteligencia emocional total que los alumnos del área $\mathrm{C}$.

8. Existen correlaciones Positivas, estadísticamente significativas entre la necesidad cognitiva y todas las áreas de la inteligencia emocional.

9. La necesidad cognitiva y las áreas de la inteligencia emocional constituyen constructos que conforman una sola dimensión.

10.En la inteligencia emocional el aspecto de la automotivación es el que obtiene la puntuación más elevada. 


\section{BIBLIOGRAFIA}

Alarcón, R. (1991). Métodos y diseños de Investigación del Comportamiento. Lima:

U.P.C.H., Fondo Editorial.

American Psychological Association (1998). Manual de Estilo de Publicaciones. México: Manual Moderno.

Anastasi, A. Urbina, S. (1998). Test Psicológicos. México: Editorial Prentice Hall

Ashforth, B.E. \& Humphrey, R.H. (1995). Emotion in the workplace: A reappraisal. Human Relations, 48(2), 97-125.

Briones, G. (1986). Métodos y Técnicas de Investigación para las Ciencias Sociales. México: Ed. Trillas.

Brockert, S. \& Braun G. (1997). Los tests de la Inteligencia Emocional. Barcelona: Ediciones Robinbook.

Brown, F. (1980). Principios de la Medición en Psicología y Educación. México: Edit. El Manual Moderno.

Cacciopo, J. T.; y, Petty R.E. (1982). The Need for Cognition. Journal of Personality and Social Psychology. $\mathrm{N}^{\circ} 42$, p. 116-131.

Cacciopo, J. T.; Petty R.E.; y, Morris, K. (1983). Effects of Need for Cognition on Message Evaluation, Recall, and Persuasion. Journal of Personality and Social Psychology. $\mathrm{N}^{\circ} 45$, p. 805-818.

Cacciopo, J. T.; y, Petty R.E. (1984). The Need for Cognition: Relationship to Attitudinal Processes. In R. McGlynn; 1. Maddux; C.

Stoltenberg; y, J. Harvey (Eds.). Social Perception in Clinical and Counseling Psychology. Lubbock, TX: Texas Tech Press.

Cacciopo, J. T.; Petty R.E.; y, Kao, Ch. (1984). The Efficient Assesment of Need for Cognition. Journal of Personality Assesment. $\mathrm{N}^{\circ} 48$, p.306-307.

Cohen, A.; Stotland, E.; y, Wolfe, D. (1955). An Experimental Investigation of Need for Cognition. Journal of Abnormal and Social Psychology. $N^{\circ}$ 51, p.291-294.

Cooper, R.\& Sawaf, A. (1997) La Inteligencia Emocional aplicada al liderazgo y a las organizaciones. Barcelona: Grupo Editorial Norma.

Delgado, Ana Esther (1997). Estudio de las Relaciones entre la Necesidad Cognitiva y las Dimensiones de la Personalidad: Extraversión, Emocionalidad y Dureza, en Estudiantes del Primer Semestre de la UNMSM. Tesis para optar el Grado de Magíster en Psicología (Mención: Psicología Educativa). UNMSM. Lima, Perú.

Escurra, L. (1991). Adaptación del Inventario de Estilos de Aprendizaje de Kolb en Estudiantes de Psicología pertenecientes a diferentes universidades de Lima Metropolitana". Tesis para optar el Título Profesional de Psicólogo. UNMSM. Lima, Perú.

García Vega, L. (1998). 100 años de la ley del Efecto a la Inteligencia Emocional.

Gardner, H. (1993). Estructuras de la mente: La teoría de las Múltiples inteligencias. México: Fondo de Cultura Económica.

Goleman, D. (1996). Inteligencia Emocional. Barcelona: Editorial Kairos.

Goleman, D. (1997). La Inteligencia Emocional de los niños. Buenos Aires: Javier Vergara Editor S.A.

Gutiérrez, M.; Bajen, M.; Sintas, F; y, Amat, M. (1993). Evaluación de la Tendencia del Esfuerzo Cognitivo. Anuario de Psicología. $\mathrm{N}^{\circ}$ 58, p. 53-67. 
Hernández, Fernández, Baptista. (1998). Metodología de la investigación. México: Mc. Graw-Hill.

Kerlinger, F. (1988) Investigación del comportamiento. México: Mac Graw-Hill

Marín, G. (1986). Consideraciones metodológicas básicas para conducir investigaciones Psicológicas en América Latina. Acta Psiquiátrica y Psicológica de América de Latina Vol. 32, Pgs. 183-192.

Mestre, J. M., Carreras M. y Guil, M (1998). Una aproximación a la evaluación de la Inteligencia Emocional como constructo teórico.

Mayer, J. D. \& Salovey, P. (1993). The Intelligence of emotional intelligence. Intelligence. Vol 17,pp.433-442.

Mayer, J. D. \& Salovey, P. (1997). Whayisemotional intelligence. In Salovey P, \& Sluyter (Eds.). Emotional development literacy and Emotional Intelligence: implications for educator. New York: Basic Books ..

Muñiz, J. (1996). Psicometría. Madrid: Ed. Universitas S. A.

Piscoya, L. (1977). Investigación Educacional Lima: INIDE.

Salovey, P. \& Mayer, J. D. (1990). Emotional intelligence. Imagination, Cognition, and Personality, 9,185-211.

Ruisel, I. (1992). Social Intelligence. Conception and methodological problems. Studia Psichologica, 34, (4-5), 281-296.

Sánchez, C. H. y Reyes, M. C. (1998). Metodología y Diseños en la Investigación Científica: Aplicados a la Psicología, Educación y Ciencias Sociales. Lima: Editorial Mantaro.

Schwab, O. P. (1980). Construct validity in organization behaviour. In B.M. Staw \& L.L. Cummings (Eds.), Research in organizational behaviour, Vol. 2. Greenwich, CT: JAI Press. Siegel, S. y Castellan N. (1995). Estadística No paramétrica: Aplicada a las ciencias de la conducta. México: Editorial Trillas.

Van Dalen y Mayer (1971). Manual de Técnica de la Investigación Educacional. Bs. As.: Ed. Paidós.

Vitor G. (1998) Inteligencia Emocional e a Escola de Educacao Emocional, Brasilia:

Escola de Educacao Emocional.

Williams, W. M. \& Stemberg, RJ. (1988). Group intelligence: Why some groups are better than others. Intelligence, 12,351-377.

Young, C. (1996). Measuring a psychological construct. Organizacional Behavior. 20,250-279. 